\title{
ON GROUPS WITH ENOUGH FINITE REPRESENTATIONS
}

D. S. PASSMAN

It has been conjectured that the group algebra $Q[G]$ of a discrete group $G$ over the rational numbers $Q$ is semi-simple. In this paper we consider irreducible representations of this algebra satisfying certain finiteness conditions and see just how much information these yield towards solving the above problem. We show in fact for a finitely generated group $G, Q[G]$ has "enough" of these representations to guarantee that it is semi-simple if and only if $G$ is a subdirect product of finite groups.

We use freely the basic results on the irreducible representations and the Jacobson radical of an algebra as found in [2].

1. The finite radicals. Let $A$ be an algebra over a field $K$ (all fields considered here are characteristic zero) and let

$$
\mathcal{F}: A \rightarrow \operatorname{Hom}_{K}(V, V)
$$

be an irreducible representation of $A$ on the module $V$.

Definition 1. $\mathcal{F}$ is said to be finite if $V$ is finite-dimensional over the center of the commuting ring of $\mathscr{F}$.

Definition $1^{*}$. $\mathcal{F}$ is said to be ${ }^{*}$ finite if $V$ is finite-dimensional over $K$.

Clearly $\mathcal{F}$ is finite if it is *finite.

Definition 2. The intersection of the kernels of all finite representations (all representations are assumed irreducible) of $A$ is called the finite radical of $A$ and is denoted by $f$ - $\operatorname{Rad} A$.

Definition 2*. The intersection of the kernels of all *finite representations of $A$ is called the ${ }^{*}$ finite radical of $A$ and is denoted by $f^{*}$-Rad $A$.

We have clearly

$$
f^{*}-\operatorname{Rad} A \supseteq f-\operatorname{Rad} A \supseteq \operatorname{Rad} A,
$$

where the last term is the Jacobson radical of $A$. If $A$ is finitedimensional over $K$ we have equality throughout.

The main results of this section are

TheOREM I. If $A$ is a finitely generated algebra over $K$ then $f^{*}$-Rad $A$ $=f-\operatorname{Rad} A$.

Received by the editors February 13, 1962 and, in revised form, March 18, 1962. 
Theorem II. If $A$ is a finitely generated algebra over $K$ and if $E$ is an extension field of $K$ then

$$
f-\operatorname{Rad}(E \otimes A)=E \otimes(f-\operatorname{Rad} A),
$$

where the tensor products are over $K$.

Let $\mathcal{F}$ be a ${ }^{*}$ finite representation of an algebra $B$. Then for all $b \in B, \mathcal{F}(b)$ can be written as a finite matrix $[\mathcal{F}(b)]$ over $K$. With respect to this we have a well defined character $\chi$ with

and we have

$$
\chi(b)=\operatorname{trace}[\mathfrak{F}(b)]
$$

Lemma 1. Kernel $\mathfrak{F}=\left\{b \in B \mid \forall b_{1} \in B, \chi\left(b b_{1}\right)=0\right\}$.

Proof. By the finiteness assumption $\mathfrak{F}(B)$ is simple so $\operatorname{ker} \mathfrak{F}$ is a maximal ideal of $B$. On the other hand, the right hand expression $H$ above is an ideal in $B$ containing the kernel. Since all fields considered here are characteristic zero $\chi(1) \neq 0$ so $1 \notin H$. Hence $H$ is a proper ideal and the result follows.

If $B=E \otimes A$ (viewed as an algebra over $E$ ) then since $\chi$ is $E$ linear we see that

$$
\operatorname{ker} \mathcal{F}=\{b \in B \mid \forall a \in A, \chi(b a)=0\} .
$$

Let $A$ now be finitely generated by the elements $a_{1}, a_{2}, \cdots, a_{t}$ over the field $K$.

Lemma 2. Let $a \in A$ and let $\mathfrak{F}$ be $a{ }^{*}$ finite representation of $E \otimes A$ such that $\mathfrak{F}(a) \neq 0$. Then there exists $a^{*}$ finite representation $\mathfrak{I}$ of $A$ with $\Im(a) \neq 0$.

Proof. Let $\chi$ be the character associated with $\mathcal{F}$ over the field $E$. By the remark after Lemma 1 there exists an element $a_{0} \in A$ with $\chi\left(a a_{0}\right) \neq 0$.

Case 1. Let $E$ be purely transcendental over $K$. Now each of the $\mathcal{F}\left(a_{i}\right)$ can be written as matrices of finite degree $m$ over $E$. The coefficients which occur are then rational functions over $K$ and so also is $\chi\left(a a_{0}\right)$. Let $p$ be the product of all the denominator polynomials in the coefficients of all the $\left[\mathfrak{F}\left(a_{i}\right)\right]$ and the numerator polynomial of $\chi\left(a a_{0}\right)$. Since $\chi\left(a a_{0}\right) \neq 0, p$ is a nonzero polynomial in a finite number of variables over $K$.

Let $x_{1}, x_{2}, \cdots, x_{j}$ be the variables which occur in all these coefficients. Since $p \neq 0$ and $K$ is infinite (having characteristic zero) there exists elements $k_{1}, k_{2}, \cdots, k_{j} \in K$ such that $p\left(k_{1}, k_{2}, \cdots, k_{j}\right) \neq 0$. The maps $x_{i} \rightarrow k_{i}$ then induce a well defined $K$ homomorphism 


$$
h: A \rightarrow \mathcal{F}(A) \rightarrow K_{m}
$$

since the $a_{i}$ generate $A$.

The result will follow if we show that the image of $a$ is not in the Jacobson radical of $h(A)$ since $h(A)$ is finite-dimensional over $K$. However, if this were not the case then $h\left(a a_{0}\right)$ would be nilpotent. But when viewed in $K_{m}$ this element has trace (which is clearly the image of $\left.\chi\left(a a_{0}\right)\right)$ not equal to zero and this is the required contradiction.

Case 2. Let $E$ be algebraic over $K$. Then the coefficients of all the $\left[F\left(a_{i}\right)\right]$ are finite in number and generate a finite field extension $E_{1}$ over $K$. Thus $\mathcal{F}(A)$ is finite-dimensional over $K$ and it suffices to show that this is semi-simple. But if this were not the case, then its radical, a nonzero nilpotent ideal, would span a nonzero nilpotent ideal in $\mathcal{F}(E \otimes A)$, a contradiction.

Case 3. Finally let $E$ be an arbitrary field extension of $K$. Let $E_{1}$ be an intermediate field generated by a transcendence base of $E / K$. Then $E / E_{1}$ is algebraic and $E_{1} / K$ is purely transcendental. By Case 2 we can find a ${ }^{*}$ finite representation $\mathcal{F}_{1}$ of $E_{1} \otimes A$ such that $\mathcal{F}_{1}(a) \neq 0$. Now by Case 1 there is a ${ }^{*}$ finite representation $\mathfrak{I}$ of $A$ with $\mathfrak{J}(a) \neq 0$ and thus the result.

Proof of Theorem I. We already have $f^{*}$-Rad $A \supseteq f$-Rad $A$. Let $a \in A$ with $a \notin f-\operatorname{Rad} A$. Then there is a finite representation $\mathcal{F}$ of $A$ with $\mathcal{F}(a) \neq 0$. If $E$ is the center of the commuting ring, then $\mathcal{F}$ can be extended in a natural way to a ${ }^{*}$ finite irreducible representation of $E \otimes A$. By Lemma 2 there is a *finite representation $\mathfrak{J}$ of $A$ with $\Im(a) \neq 0$. Thus $a \notin f^{*}$ - $\operatorname{Rad} A$ and this yields the reverse inclusion.

Proof of Theorem II. Since the $f$ and $f^{*}$ radicals are the same, we will prove this result for the $f^{*}$ radical instead. If $a \in f^{*}$-Red $A$ then by Lemma 2 there are no *finite representations $\mathcal{F}$ of $E \otimes A$ with $\mathcal{F}(a) \neq 0$. Hence $a \in f^{*}-\operatorname{Rad}(E \otimes A)$. Thus $f^{*}-\operatorname{Rad}(E \otimes A)$ $\supseteq E \otimes f^{*}$-Rad $A$.

Conversely let $b=e_{1} \alpha_{1}+e_{2} \alpha_{2}+\cdots+e_{n} \alpha_{n}$ be an element of $f^{*}$ - $\operatorname{Rad}(E \otimes A)$ where the $e_{i}$ are elements of $E$ linearly independent over $K$ and the $\alpha_{i} \in A$. Let $\mathcal{F}$ be a ${ }^{*}$ finite representation of $A$. Then $\mathfrak{F}(A)$ is a simple finite-dimensional algebra over $K$ and thus $E \otimes \mathfrak{F}(A)$ is semi-simple. Hence under the representation

$$
E \otimes A \rightarrow E \otimes F(A)
$$

$b$ must map into zero. Thus

$$
e_{1}\left[F\left(\alpha_{1}\right)\right]+\cdots+e_{n}\left[F\left(\alpha_{n}\right)\right]=0 .
$$

Since each matrix $\left[\mathcal{F}\left(\alpha_{i}\right)\right]$ has coefficients in $K$, by the linear inde- 
pendence of the $e_{i}$ we have for all $i, F\left(\alpha_{i}\right)=0$. Since this is true for all *finite representations of $A$, it follows that each $\alpha_{i} \in f^{*}-\operatorname{Rad} A$ and we have the reverse inclusion.

2. Group algebras. If $G$ is a finitely generated group, then these elements and their inverses will generate $Q[G]$ over $Q$. Hence the group algebra $Q[G]$ is finitely generated. We now determine its finite radical.

TheOREM III. Let $G$ be a finitely generated group. The following are equivalent:

(i) $f-\operatorname{Rad} Q[G]=(0)$,

(ii) $f^{*}$ - $\operatorname{Rad} Q[G]=(0)$,

(iii) $G$ is a subdirect product of finite groups.

TheORem IV. Let $G$ be a finitely generated group and let $H$ be the intersection of all normal subyroups of finite index in $G$. Then $f-\operatorname{Rad} Q[G]$ is the smallest ideal containing all the elements of the form $1-h$ with $h \in H$.

We first need the following

Lemma 3. Let $g \in G$ be such that $1-g \notin f^{*}$-Rad $Q[G]$. Then there exists a normal subgroup $N$ of finite index in $G$ with $g \notin N$.

Proof. Since $1-g \notin f^{*}$ - $\operatorname{Rad} Q[G]$, there is a *finite representation $\mathcal{F}$ with $\mathcal{F}(1) \neq F(g)$. Now $\mathcal{F}(G)$ is a finitely generated multiplicative subgroup of $Q_{m}$ the $m \times m$ matrices over $Q$. Let $\pi$ denote the set of primes (in the rational integers) which occur in the denominators of the coefficients of the generators and their inverses. Then $\pi$ is of course a finite set.

For any prime $p \notin \pi, \mathcal{F}(G) \subseteq\left(Z_{p}\right)_{m}$, the latter being the $m \times m$ matrices over the integers $Z$ localized at $p$. The homomorphism

$$
Z_{p} \rightarrow Z_{p} / p Z_{p}=Z / p Z
$$

then induces a homomorphism $\theta_{p}$ of $\mathcal{F}(G)$ onto a finite group. If we assume now that $p$ does not divide the numerator of some coefficient of $\mathfrak{F}(1-g) \neq 0$ then $g \notin N$ where $N$ is the kernel in $G$ of $\theta_{p} \circ \mathcal{F}$. Since $N$ has finite index the result follows.

PROOF OF THEOREM III.

(i) $\rightarrow$ (ii). This follows immediately from Theorem I.

(ii) $\rightarrow$ (iii). Let $g \neq 1$ be an element of $G$. Since $1-g \notin f^{*}$-Rad $Q[G]$, there exists a normal subgroup $N$ of finite index with $g \notin N$ by Lemma 3 . Hence the intersection of all such subgroups is (1) and the result follows. 
(iii) $\rightarrow($ i). If $f-\operatorname{Rad} Q[G] \neq(0)$ then by multiplying some element in the ideal by a suitable group and field element we can assume that

$$
x=1+q_{1} g_{1}+q_{2} g_{2}+\cdots+q_{j} g_{j} \in f-\operatorname{Rad} Q[G],
$$

where the $q_{i} \in Q$ and the $g_{i} \in G$ with all $g_{i}$ distinct and not equal to the identity.

Since $G$ is a subdirect product of finite groups there is a normal subgroup $N$ of finite index in $G$ with $g_{i} \notin N$ for all $i$. The natural map $Q[G] \rightarrow Q[G / N]$ maps $Q[G]$ onto a semi-simple finite-dimensional algebra. Thus $f$ - $\operatorname{Rad} Q[G]$ is contained in the kernel of this homomorphism. In particular the image of $x$ is zero. This is the required contradiction since by our choice of $N$ the identity term will not be cancelled.

We remark that (iii) $\rightarrow$ (i) is a special case of a result of Villamayor in [4]. Our proof also does not require that $G$ is finitely generated and it yields the additional result that the irreducible representations of the algebras $Q[G / N]$ viewed as representations of $Q[G]$ form a complete set of representations for the group algebra.

Proof of Theorem IV. This is essentially a corollary of the above. Let $I$ be the ideal of $Q[G]$ spanned by the elements $1-h$ with $h \in H$. Then $I$ is the kernel of the homomorphism $Q[G] \rightarrow Q[G / H]$. Since $G / H$ is a subdirect product of finite groups we have $f$ - $\operatorname{Rad} Q[G / H]$ $=(0)$. But the map is surjective so this clearly implies that $f$ - $\operatorname{Rad}$ $Q[G] \subseteq I$.

On the other hand, by Lemma 3, we see that for all $h \in H$ we have $1-h \in f$ - $\operatorname{Rad} Q[G]$ so we obtain the reverse inclusion.

The following result allows finite methods to be used in studying groups having all absolutely irreducible representations finite.

TheOREM V. Let $G$ be a finitely generated group. If all the irreducible representations of $C[G]$, the group algebra over the complex numbers, are finite, then $G$ is a subdirect product of finite groups.

Proof. By assumption $f$ - $\operatorname{Rad} C[G]=\operatorname{Rad} C[G]$ and the latter is zero by Theorem 5.2 of $[3]$. Since $C[G]=C \otimes Q[G]$, Theorem II yields $f$ - $\operatorname{Rad} Q[G]=(0)$. By Theorem III the result follows.

Finally we mention that Fuchs-Rabinowitsch gives an example in [1] of a finitely presented group for which $f$ - $\operatorname{Rad} Q[G]$ is not zero. It is actually easier using his same trick to show that the group is not a subdirect product of finite groups.

\section{REFERENCES}

1. D. J. Fuchs-Rabinowitsch, Über eine Gruppe mit endlichvielen Erzeugenden und 
Relationen, die keine Isomorphedarstellung durch Matrizen von endlicher Ordnung zulasst, Dokl. Akad. Nauk. SSSR 27 (1940), 425-426.

2. N. Jacobson, Structure of rings, Amer. Math. Soc. Colloq. Publ. Vol. 37, Amer. Math. Soc., Providence, R. I., 1956.

3. C. Rickart, Uniqueness of norm in Banach algebras, Ann. of Math. (2) 51 (1950), 615-628.

4. O. E. Villamayor, On the semisimplicity of group algebras. II, Proc. Amer. Math. Soc. 10 (1959), 27-31.

HARVARD UNIVERSITY

\section{CERTAIN PROBLEMS OF DIFFERENTIABLE IMBEDDING}

\section{YASURÔ TOMONAGA}

1. The differentiable imbedding of the complex projective spaces has been studied by many authors $[1 ; 3 ; 4 ; 5]$. In this note we shall deal with the nonimbeddability of the submanifolds of a complex projective space. It was studied in [6] in a particular case.

We denote by $P_{n}(c)$ the complex projective space of complex dimension $n$. Let $V_{2 n-2}$ be a differentiable compact orientable submanifold of $P_{n}(c)$ corresponding to a cohomology class $v \in H^{2}\left(P_{n}(c), Z\right)$. Then the Pontrjagin class of $V_{2 n-2}$ is determined as follows [2]:

$$
\begin{gathered}
j: V_{2 n-2} \rightarrow P_{n}(c) \\
1+p_{1}\left(V_{2 n-2}\right)+p_{2}\left(V_{2 n-2}\right)+\cdots \\
=j^{*}\left[\left(1+p_{1}\left(P_{n}(c)\right)+p_{2}\left(P_{n}(c)\right)+\cdots\right)\left(1+v^{2}\right)^{-1}\right]
\end{gathered}
$$

where $p_{i}$ denotes the Pontrjagin class of the dimension $4 i$. We put as follows:

$$
\begin{aligned}
p & =\sum_{k \geq 0}(-1)^{k} p_{k}=\prod_{\alpha}\left(1-\gamma_{\alpha}\right), \\
p & =\sum_{k \geq 0} p_{k}=\prod_{\alpha}\left(1-\gamma_{\alpha}\right)^{-1}, \\
p \cdot \bar{p} & =1 .
\end{aligned}
$$

In the case of $P_{n}(c)$ we have

$$
\begin{aligned}
& p=\left(1-g_{n}^{2}\right)^{n+1}, \quad g_{n} \in H^{2}\left(P_{n}(c), Z\right), \\
& \bar{p}=\left(1-g_{n}^{2}\right)^{-n-1} .
\end{aligned}
$$

Received by the editors June 8, 1962. 